\title{
Katrin Rohr-Suchalla
}

\section{VOB

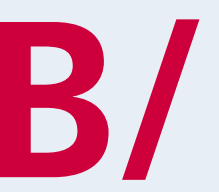 \\ asiswissen für
aufachleute}

2., überarbeitete und aktualisierte Auflage

Fraunhofer IRB Verlag 
Katrin Rohr-Suchalla

VOB/B - Basiswissen für Baufachleute 
Katrin Rohr-Suchalla

\section{VOB/B - Basiswissen für Baufachleute}

2., überarbeitete Auflage

Fraunhofer IRB Verlag 
Bibliografische Information der Deutschen Nationalbibliothek

Die Deutsche Nationalbibliothek verzeichnet diese Publikation in der Deutschen Nationalbibliografie; detaillierte bibliografische Daten sind im Internet über <http:/ / www.dnb.de> abrufbar.

ISBN (Print): 978-3-8167-8675-7

ISBN (E-Book): 978-3-8167-8774-7

Layout: Mitterweger \& Partner, Plankstadt

Satz: Laura Schweiger

Umschlaggestaltung: Martin Kjer, Fraunhofer IRB Verlag

Druck: Druckerei \& Verlag, Steinmeier, Deiningen

C) by Fraunhofer IRB Verlag, 2013

Fraunhofer-Informationszentrum Raum und Bau IRB

Postfach 8004 69, 70504 Stuttgart

Telefon (0711) 970-2500

Telefax (0711) 970-2508

E-Mail irb@irb.fraunhofer.de

http://www.baufachinformation.de

Alle Rechte vorbehalten

Dieses Werk ist einschließlich aller seiner Teile urheberrechtlich geschützt. Jede Verwertung, die über die engen Grenzen des Urheberrechtsgesetzes hinausgeht, ist ohne schriftliche Zustimmung des Fraunhofer IRB Verlages unzulässig und strafbar. Dies gilt insbesondere für Vervielfältigungen, Übersetzungen, Mikroverfilmungen sowie die Speicherung in elektronischen Systemen.

Die Wiedergabe von Warenbezeichnungen und Handelsnamen in diesem Buch berechtigt nicht zu der Annahme, dass solche Bezeichnungen im Sinne der Warenzeichen- und Markenschutz-Gesetzgebung als frei zu betrachten wären und deshalb von jedermann benutzt werden dürften.

Sollte in diesem Werk direkt oder indirekt auf Gesetze, Vorschriften oder Richtlinien (z.B. DIN, VDI, VDE) Bezug genommen oder aus ihnen zitiert werden, kann der Verlag keine Gewähr für Richtigkeit, Vollständigkeit oder Aktualität übernehmen. Es empfiehlt sich, gegebenenfalls für die eigenen Arbeiten die vollständigen Vorschriften oder Richtlinien in der jeweils gültigen Fassung hinzuzuziehen. 


\section{Inhaltsverzeichnis}

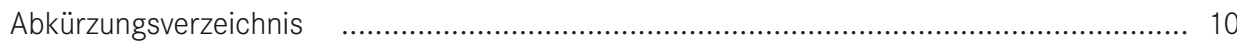

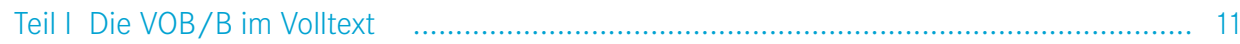

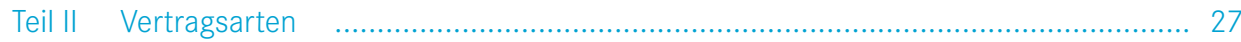

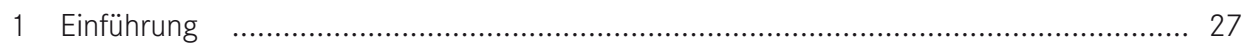

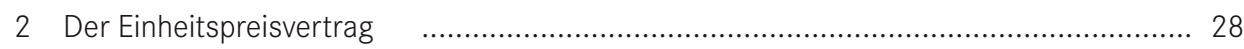

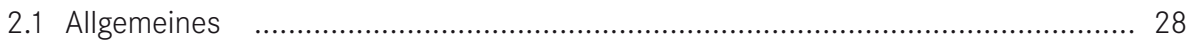

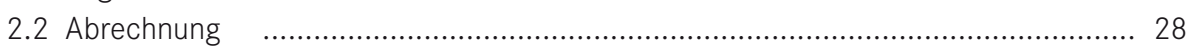

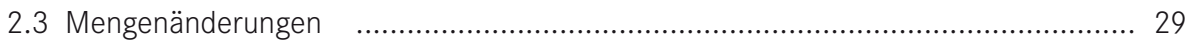

2.4 Nebenleistungen/Besondere Leistungen ….................................................. 33

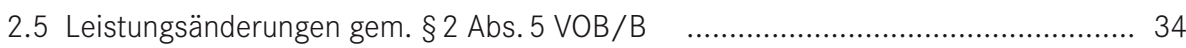

2.6 Zusätzliche Leistungen gem. § 2 Abs. 6 VOB/B ................................................ 38

2.7 Gegenüberstellung der Leistungsänderung und zusätzlicher Leistungen ............... 40

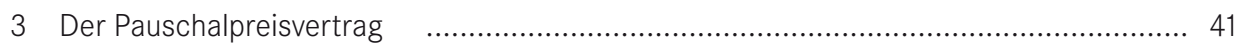

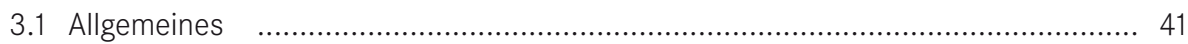

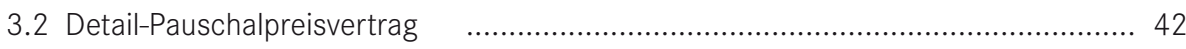

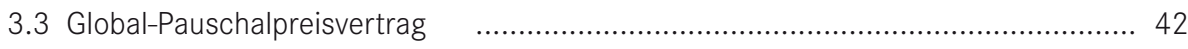

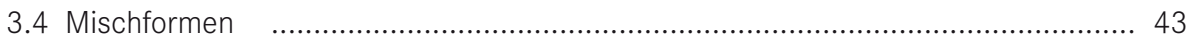

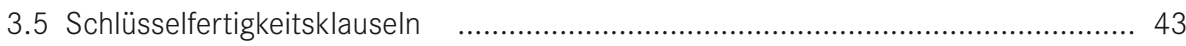

3.6 Komplettheitsklauseln/Vollständigkeitsklauseln/Frage der Wirksamkeit ............ 43

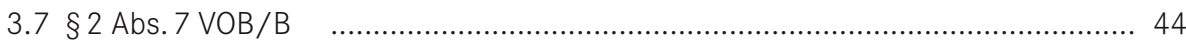

3.8 Leistungsänderung gem. § 2 Abs. 5 VOB/B …............................................... 45

3.9 Zusatzleistungen gem. § 2 Abs. 6 VOB/B .......................................................... 46

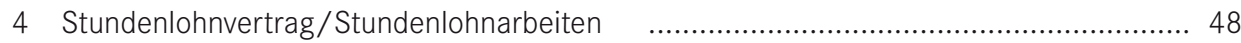

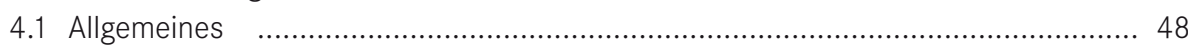

4.2 Ausdrückliche Vereinbarung gem. § 2 Abs. 10 VOB/B _..................................... 48

4.3 Vereinbarung und Abrechnung der Höhe nach (§15 VOB/B) ............................ 49

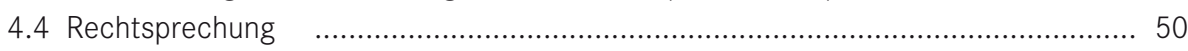

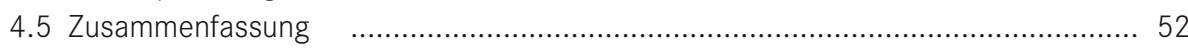

5 Selbstkostenerstattungsvertrag …................................................................. 53

Teil III Allgemeine Geschäftsbedingungen im Bauvertrag . ...................................... 55

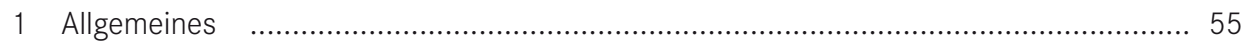

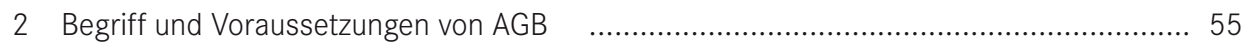

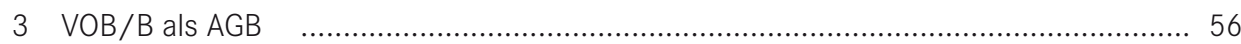

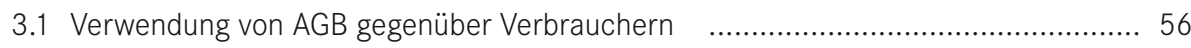

3.2 Verwendung von AGB gegenüber Unternehmern $\quad$........................................... 57

3.3 Folgen der Unwirksamkeit einer Klausel ...................................................... 57

3.4 Rechtsprechung zu ausgewählten typischen Klauseln .................................... 58 


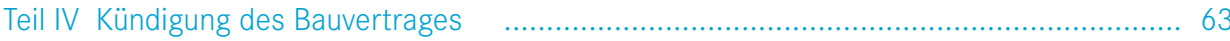

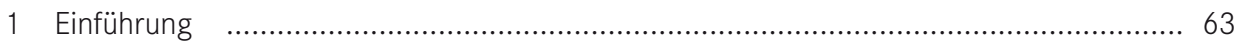

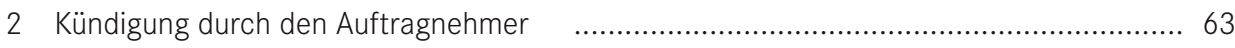

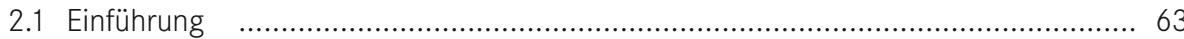

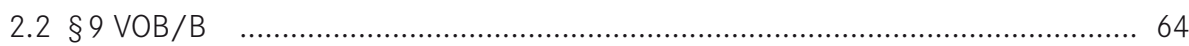

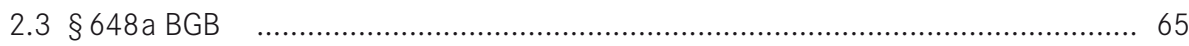

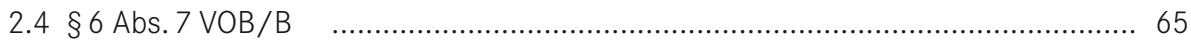

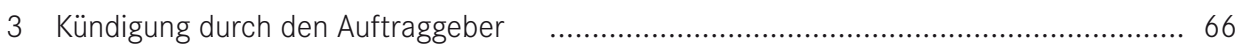

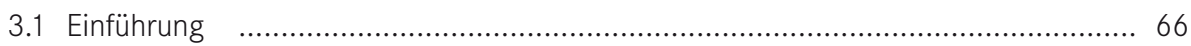

3.2 Einfache Kündigung durch den Auftraggeber ......................................................6 66

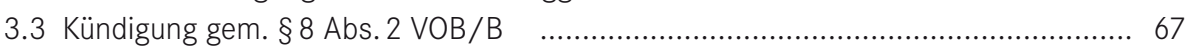

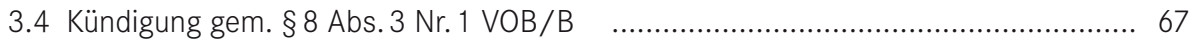

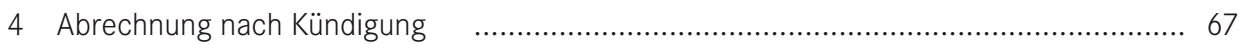

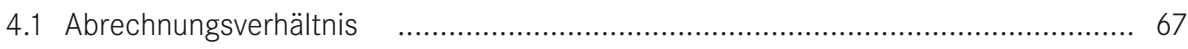

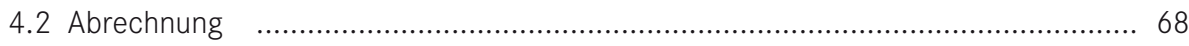

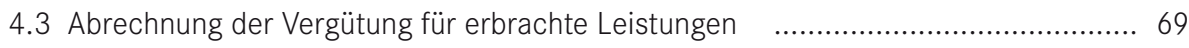

4.4 Abrechnung der Vergütung für die noch nicht erbrachten Leistungen $\quad$.................. 70

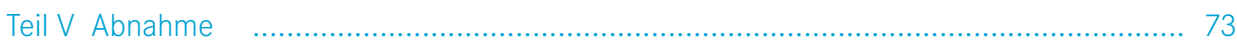

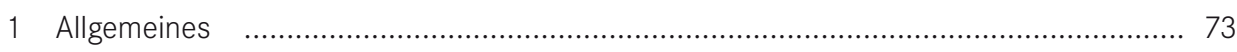

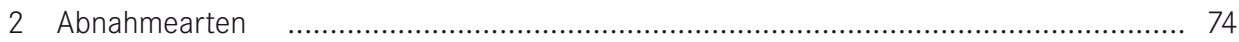

2.1 Abnahmeverlangen des Auftragnehmers ( $\S 12$ Abs. 1 VOB/B) $\quad$............................ 74

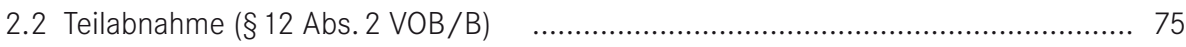

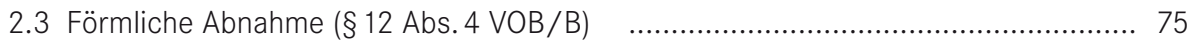

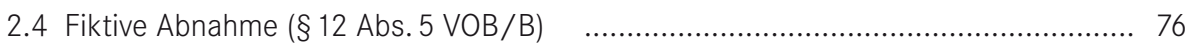

2.5 Abnahmefiktion gemäß § 640 Abs. 1 Satz 3 BGB ............................................... 76

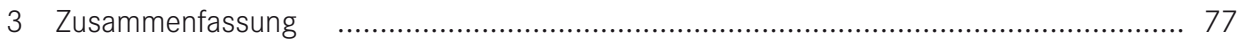

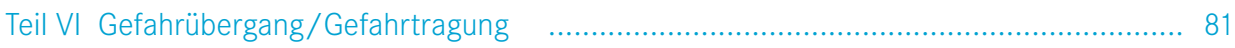

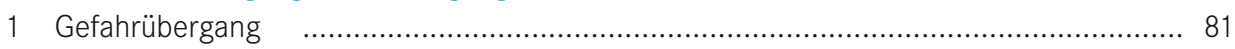

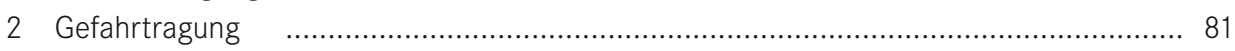

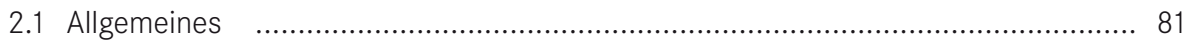

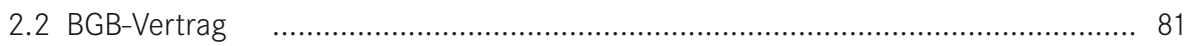

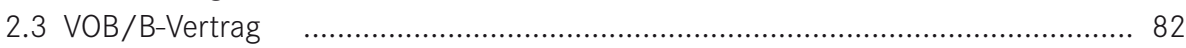

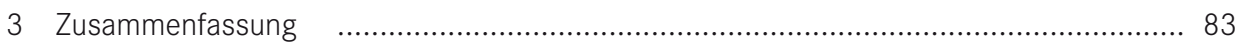

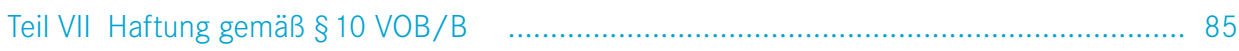

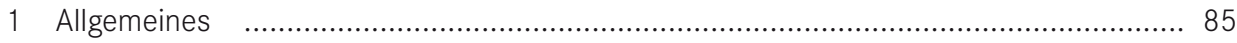

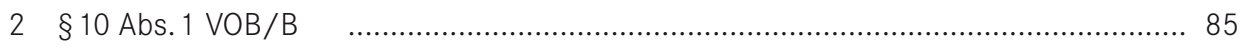

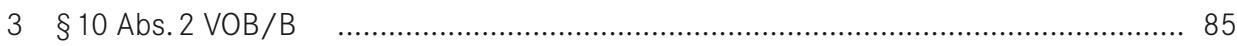

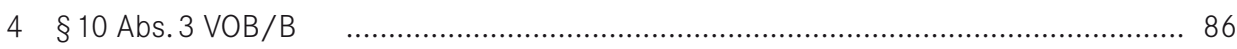

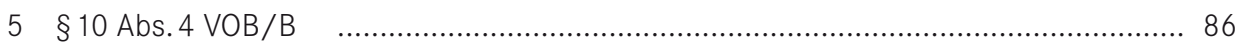

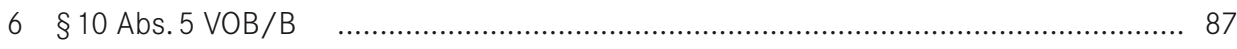


$7 \S 10$ Abs. 6 VOB/B 87

8 Zusammenfassung

Teil VIII Gewährleistung nach der VOB /B ............................................................ 89

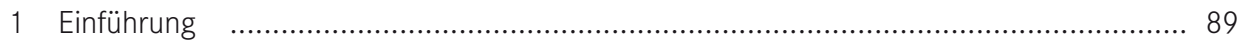

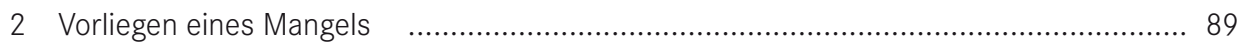

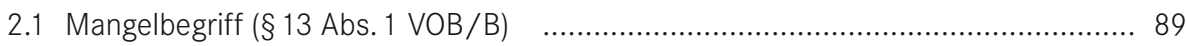

2.1.1 Vertraglich vereinbarte Beschaffenheit ............................................... 89

2.1.2 Verstoß gegen die anerkannten Regeln der Technik ............................ 89

2.2 Haftungsbefreiung nach $\S 13$ Abs. 3 VOB/B .................................................... 90

2.2.1 Voraussetzungen des $\S 13$ Abs. 3 VOB/B .............................................. 90

2.2.2 Mangel der Leistungsbeschreibung ...................................................... 91

2.2.3 Mangel durch Anordnung des Auftraggebers .......................................... 91

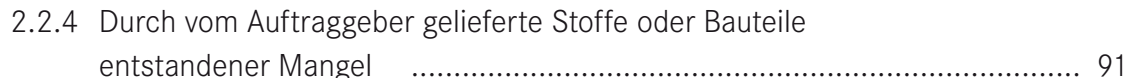

2.2.5 Durch vom Auftraggeber vorgeschriebene Stoffe oder Bauteile
entstandener Mangel

2.2.6 Durch die Vorleistung eines anderen Unternehmens entstandener Mangel ….................................................................... 91

2.2.7 Prüfungs- und Hinweispflicht gem. § 4 Abs. 3 VOB/B ............................ 92

2.2.8 Darlegungs- und Beweislast ….......................................................... 93

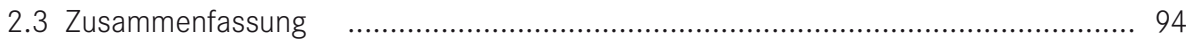

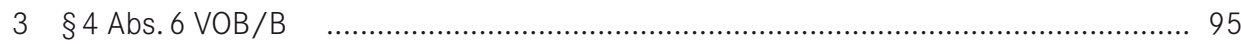

4 Mangelbeseitigungsansprüche vor Abnahme gem. §4 Abs. 7 VOB/B …...................... 95

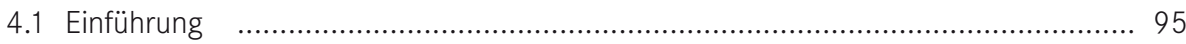

4.2 Der Mangelbeseitigungsanspruch gem. § 4 Abs. 7 VOB/B ................................ 96

4.3 Sonstige Ansprüche des Auftraggebers nach § 4 Abs. 7 VOB /B .......................... 97

4.3.1 Anspruchsvoraussetzungen ........................................................... 97

4.3.2 Rechtsfolgen ............................................................................. 98

5 Gewährleistungsansprüche nach $\S 13$ Abs. 5-7 VOB/B …................................... 100

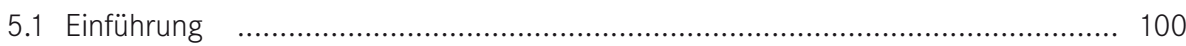

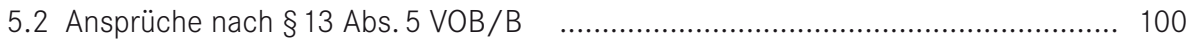

5.2.1 Mangelbeseitigungsaufforderung …................................................ 100

5.2.2 Selbsthilferecht des Auftraggebers gem. $\S 13$ Abs. 5 Nr. 2 VOB/B $\quad$........... 102

5.3 Minderungsanspruch gem. $\S 13$ Abs. 6 VOB/B .............................................. 102

5.4 Zusätzlicher Schadensersatzanspruch gem. § 13 Abs. 7 VOB/B _...................... 103

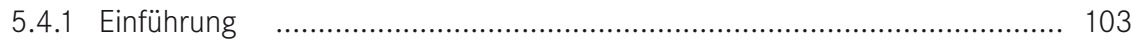

5.4.2 Kleiner Schadensersatzanspruch (§13 Abs. 7 Nr. 3 S. 1 VOB/B) _............. 103

5.4.3 Großer Schadensersatzanspruch gem. § 13 Abs. 7 Nr. 3 S. 2 VOB/B ....... 105

5.4.4 Zusammenfassung .................................................................. 106

6 Vorteilsausgleichung und Berücksichtigung eines Mitverschuldens

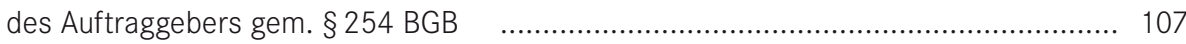

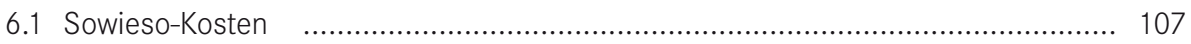


6.2 So genannter $\rrbracket$ Abzug Neu für Alt»

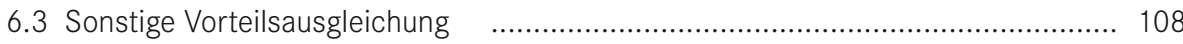

6.4 Berücksichtigung eines Mitverschuldens und Schadens
minderungspflicht des Auftraggebers gemäß §254 BGB $\ldots \ldots \ldots \ldots \ldots \ldots \ldots \ldots \ldots \ldots \ldots \ldots \ldots \ldots \ldots \ldots \ldots \ldots$

6.5 Berücksichtigung einer Vorteilsausgleichung und eines

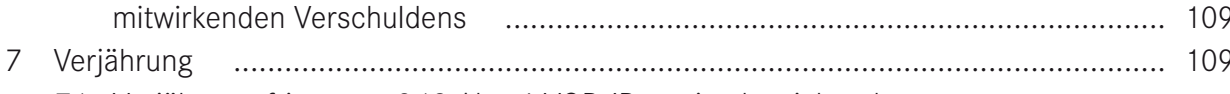

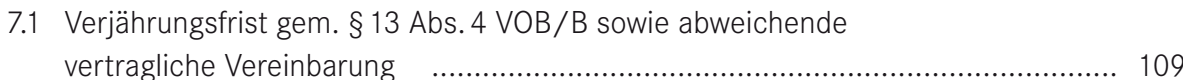

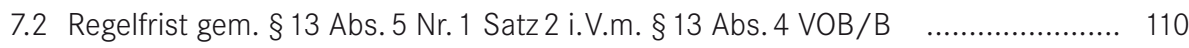

7.3 Hemmungstatbestände …........................................................................... 111

7.4 Aufrechnung und Zurückbehaltungsrecht nach Eintritt der Verjährung .............. 113

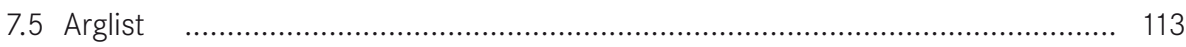

Teil IX Vertragliche und gesetzliche Sicherheiten im Baurecht $\quad$.................................. 115

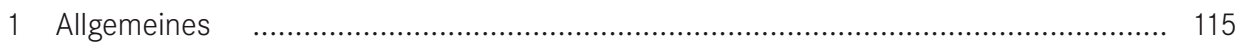

2 Sicherheiten für den Auftragnehmer …........................................................... 115

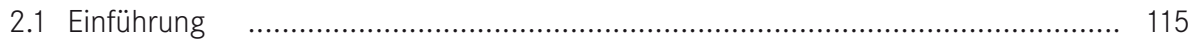

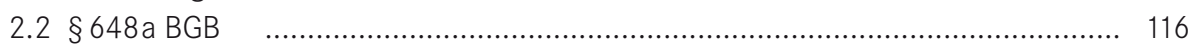

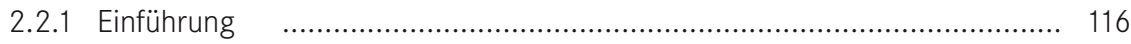

2.2.2 Anspruchsberechtigte gem. $\S 648$ a Abs. 1 BGB …................................... 117

2.2.3 Höhe der Sicherheitsleistung …......................................................... 117

2.2.4 Angemessene Frist …............................................................... 120

2.2.5 Sicherungsmittel …..................................................................... 120

2.2.6 Kein Ausschluss gem. § 648 a Abs. 6 BGB …...................................... 121

2.2.7 Unwirksamkeit von entgegenstehenden Vereinbarungen $\quad$....................... 121

2.2.8 Rechtsfolgen einer nicht rechtzeitig oder nicht gestellten Sicherheit $\quad$....... 121

2.2.9 Avalkosten ............................................................................... 121

2.2.10 Anwendbarkeit des $\S 648$ a BGB nach Abnahme .................................. 122

2.2.11 Zusammenfassung ….............................................................. 123

2.3 Eintragung einer Sicherungshypothek gem. § 648 BGB …............................. 124

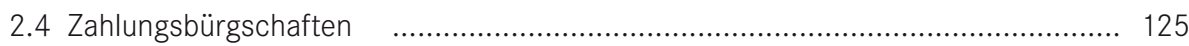

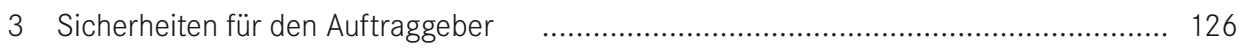

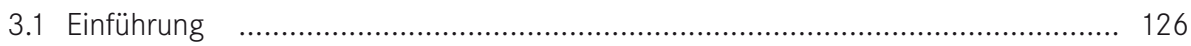

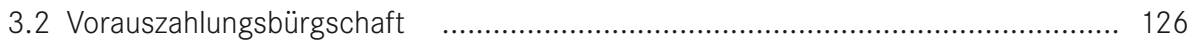

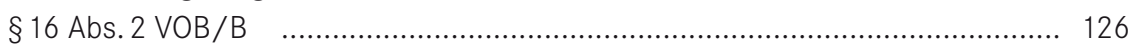

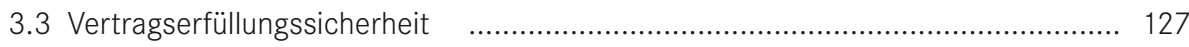

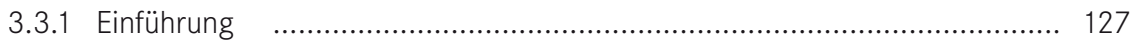

3.3.2 Arten der Sicherheitsleistung nach Wahl des Auftragnehmers ............... 128

3.3.3 Beschränkung der Austauschmöglichkeit …....................................... 129

3.3.4 Besonderheiten beim Austausch …................................................... 130

3.3.5 Zweck und Höhe der Sicherheitsleistung .......................................... 130

3.3.6 Rückgabe der Sicherheit f......................................................... 131

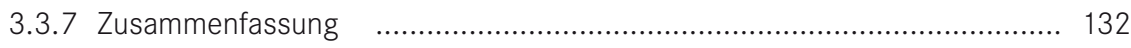




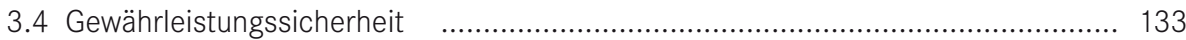

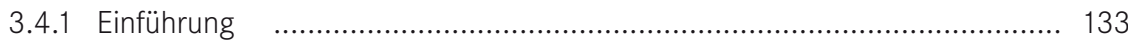

3.4.2 Höhe der Gewährleistungssicherheit .............................................. 133

3.4.3 Verpflichtung zur Stellung einer Bürgschaft auf erstes Anfordern ........... 133

3.4.4 Umfang der Haftung ................................................................. 134

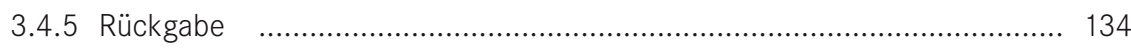

3.3.6 Zusammenfassung …................................................................. 136

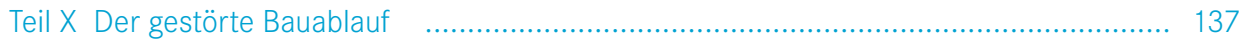

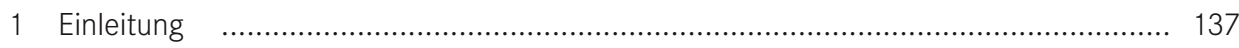

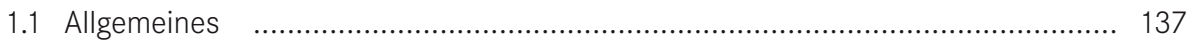

1.2 Störungen/Behinderungen des Bauablaufs ................................................. 138

2.1 Problematik und Schwierigkeit des gestörten Bauablaufs ................................. 139

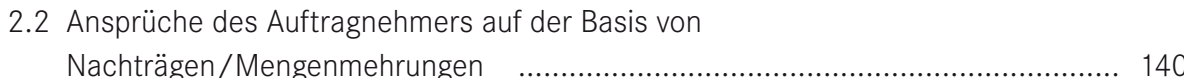

2.3 Beschleunigungsanordnung/Anordnung in zeitlicher Hinsicht gem. § 2 Abs. 5 VOB/B …................................................... 140

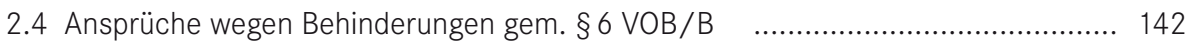

2.4.1 Voraussetzungen .......................................................................... 142

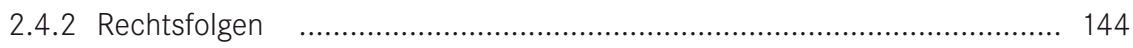

2.4.3 Bauzeitverlängerungsanspruch/Fortführungsverpflichtung .................... 144

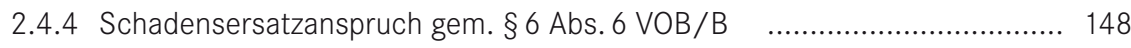

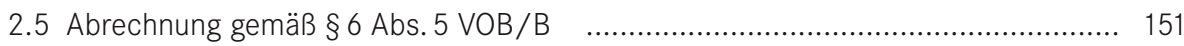

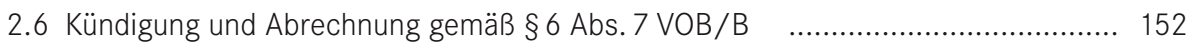

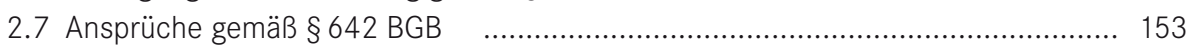

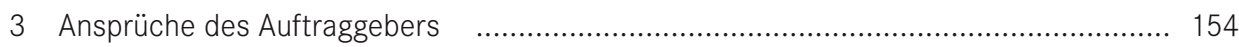

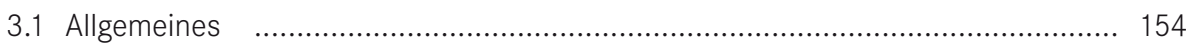

3.2 Übersichtsdarstellung der Ansprüche des Auftraggebers ................................ 154

3.3 Voraussetzungen und Rechtsfolgen von Ansprüchen nach § 5 Abs. 4 VOB/B $\quad$..... 156

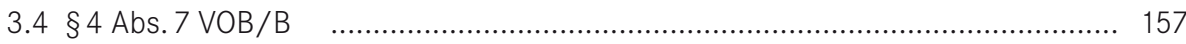

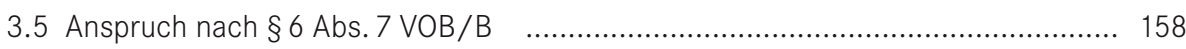

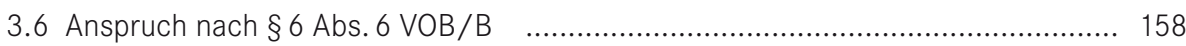

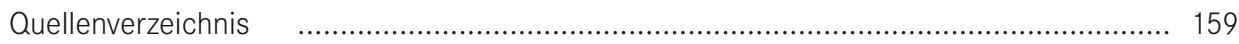

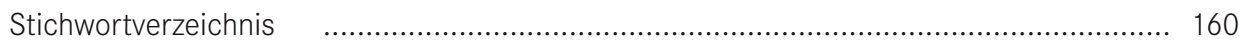




\section{Abkürzungsverzeichnis}

$\begin{array}{ll}\text { AG } & \text { Auftraggeber } \\ \text { AGB } & \text { Allgemeine Geschäftsbedingungen } \\ \text { AN } & \text { Auftragnehmer } \\ \text { BGB } & \text { Bürgerliches Gesetzbuch } \\ \text { BGH } & \text { Bundesgerichtshof } \\ \text { d.h. } & \text { das heißt } \\ \text { DIN } & \text { Deutsche Industrienorm } \\ \text { GoA } & \text { Geschäftsführung ohne Auftrag } \\ \text { GU } & \text { Generalunternehmer } \\ \text { Nr. } & \text { Nummer } \\ \text { NU } & \text { Nachunternehmer } \\ \text { OLG } & \text { Oberlandesgericht } \\ \text { RG } & \text { Reichsgericht } \\ \text { TA Luft } & \text { Technische Anleitung zur Reinhaltung der Luft } \\ \text { UVV } & \text { Unfallverhütungsvorschriften } \\ \text { VOB/A/B/C } & \text { Verdingungsordnung für Bauleistungen Teil A, Teil B und Teil C } \\ \text { z.B. } & \text { zum Beispiel } \\ \text { ZPO } & \text { Zivilprozessordnung }\end{array}$

